\title{
Guideline for oral healthcare of adults with Huntington's disease
}

\author{
Graham Manley', Helen Lane1, Annette Carlsson², Bitte Ahlborg², Åsa \\ Mårtensson², Monica B Nilsson², Sheila A Simpson ${ }^{3,4}$ \& Daniela Rae ${ }^{* 3,4}$; \\ On behalf of the contributing members of the European Huntington's \\ Disease Networks Standards of Care Dental Care Group
}

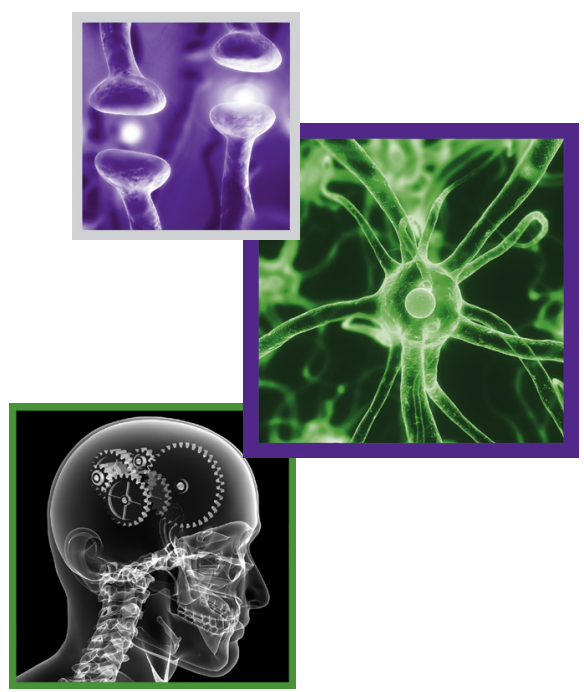

- A preventive dentistry regime should be implemented at the earliest possible opportunity and
maintained throughout development of the condition. The use of high fluoride toothpaste is
essential.
Discuss with dieticians their recommendations for use of highly cariogenic foods. Work closely with
all professional and nonprofessional groups concerned with care of the person with Huntington's
disease.
In the early stage of onset of the condition, consider carefully before providing advanced
conservative treatment (i.e., crowns, bridges, implants). In the later stages, maintenance of complex
restorative dentistry may present real problems for dentists and caregivers. This may be a difficult
issue for patients and/or caregivers to accept and will depend upon each individual clinical situation.
The use of intravenous conscious sedation is an extremely valuable tool for dentists when treating
people in the mid and late stages of the condition. It may avoid complicated general anesthetic hospital
admissions.
In the case of a recurrent lip trauma from choreic mouth movements discuss with caregivers
frequency and severity of event and options for prevention, using a mouth guard (if appropriate and
safe) or extractions if necessary.
As a dentist with experience of treating people with Huntington's disease you must consider it an
essential responsibility to become involved in training other colleagues.
Always assume (unless told or from experience) that the patient with Huntington's disease
understands and can communicate. Respect his/her wishes even if they do not comply with what
you as a dentist considers to be in the interest of good oral health.

SUMMARY These guidelines present an overall strategy for oral healthcare based on the principles of achieving a disease-free, pain-free and safe mouth. The standards of care referred to in the document seek to provide guidelines for the care and treatment that is no less a standard provided for an individual that does not have this condition. Such care and treatment will take into account the health and safety of each individual within the context of their condition. Particular emphasis is placed on dental professionals working within a multidisciplinary team, focusing on prevention of oral disease and providing treatment appropriate to the various stages of the progression of this condition. It is intended that by providing and subsequently promoting these guidelines, it will focus the dental professions work on this condition and the implementation of good care for people with Huntington's disease.

'The Royal Hospital for Neurodisability, London, UK

${ }^{2}$ Mun-H-Center, Goteborg, Sweden

${ }^{3}$ Department of Clinical Genetics, NHS Grampian, Aberdeen, Scotland, UK

${ }^{4}$ University of Aberdeen, Aberdeen, AB25 2ZA Scotland, UK

*Author for correspondence: Tel.: +44 1224552 120; d.rae@nhs.net

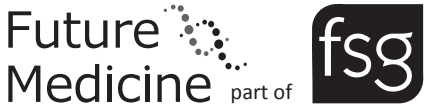


Huntington's disease (HD) is an adult-onset, familial disorder that can affect both men and women [1]. Symptom onset usually occurs by the age of 40 years, but this can be very variable. Age of onset is related to the size of the mutation, which is an unstable expansion of a CAG sequence in the gene [2]. The classical triad of clinical features includes a movement disorder, cognitive impairment and personality and psychiatric disorder. These features cause complex management problems, which adversely affects oral health. For example, the provision of mouth care and dental care may be physically limited by the movement disorder and, furthermore, oral hygiene may be neglected or declined. Described by George Huntington in 1873 , this disease continues to devastate families and perplex the clinicians who care for them.

When working with people with HD and their families it is important to provide a cohesive and multidisciplinary service. The disease affects many different aspects of the individual's abilities and they require assessment by appropriately trained healthcare professionals. No one professional will have all the skills needed to help any one individual. It is therefore of importance that the service providers take a multidisciplinary approach to HD in order to identify the best way to assist individual patients by taking into account their differing needs.

The provision of oral healthcare for people with $\mathrm{HD}$ is dependent on the dental team working together with various agencies that provide care [3]. Interdisciplinary working is essential to the effective management of people with HD. Team members will include general medical practitioners, neurologists, psychiatrists, neuropsychologists, specialist nurses, genetic counselors, social workers, care managers, palliative care consultants, dieticians, physiotherapists, occupational therapists and speech and language therapists.

The European Huntington's Disease Network (EHDN) was formed in 2003, to provide a platform for clinicians and families to work together to find a cure for Huntington's disease. In addition, working groups were created to address various themes; one of which is the Standards of Care ( $\mathrm{SoC}$ ). Within the Standards of Care Group, as part of the EHDN [101] the dental section has proposed these guidelines based on the currently available evidence as well as expert consensus on the provision of oral healthcare for individuals with HD. It is clear that much work must be undertaken in order to clarify some of the aspects of dental management.

\section{Methods}

A systematic literature search was performed electronically using Embase, Ovid MEDLINE and Ovid MEDLINE ${ }^{\circledR}$ In-Process \& Other Non-Indexed Citations.

There is very limited literature about the oral health of people with HD. The majority of literature is confined to individual patient case reports and their management strategies. Few of the papers identified proved relevant and none were above evidence grade IV (expert opinion).

Interestingly, a review of the literature focusing on multidisciplinary working in HD found only one paper that included dentistry in the multidisciplinary team [4]. A further paper states that "ideally a dentist should be part of the multidisciplinary care team with progressive neurogenic disorder from diagnosis, so that oral care can be planned throughout the disease process and does not become crisis management in the final phase of the condition" [5]. A chapter within a multidisciplinary publication considers oral healthcare [6]. This presents a literature review rather than discussing the issues involved in including this aspect of care within multidisciplinary work.

\section{Huntington's disease \& oral health}

There are no innate dental features of HD that make an individual more susceptible to dental caries or periodontal disease [7]. However, a study of the oral health of a group of individuals with mid-late stage HD in a specialized residential care facility showed that this group of individuals had significantly more missing and decayed teeth, fewer sound and restored teeth and fewer pairs of contacting posterior teeth when compared with the general population [8].

There are several contributory factors that may detrimentally affect the oral health of people with HD. These are:

- Nutritional factors

- Percutaneous endoscopic gastrostomy (PEG)

- Medication

- Oral hygiene

- Access to and provision of dental care

- Additional dental considerations 


\section{- Nutritional factors}

The 1945-1953 Vipeholm study is one of the largest and most influential single studies investigating the association between sugar consumption and dental caries [9]. It is notable that such a study which concluded results that have proved essential to the prevention of dental caries involved manipulation and 'use' of adults with learning disability in a residential institute. It concluded that consumption of sugary food and drinks both between meals and at meals is associated with a large caries increment. For ethical reasons, this study has never been repeated but the conclusions have been ratified by more recent national reports [10-12].

Several dietary factors are associated with caries incidence:

- Amount of fermentable carbohydrate consumed

- Sugar concentration of food

- Physical form of carbohydrate

- Oral retentiveness (length of time teeth are exposed to decreased plaque $\mathrm{pH}$ )

- Frequency of eating meals and snacks

- Length of interval between eating

- Sequence of food consumption

People with HD are also known to retain food within the mouth after swallowing [13,14], which will also increase the likelihood of dental caries. It is thought that the bolus retention is predominately due to dysphagia, but may also be due to deficient purposeful lingual searching activity [15]. Thickened fluids can also be difficult to clear from the mouth [7], which increases the caries risk.

In order to maintain and/or increase the calorific intake of a person with HD, many dietitians recommend eating frequent snacks high in calories and to consume lots of sugar or sugary substances, such as honey, jam, syrup and treacle $[102,103]$. It is widely accepted that a high frequency of consumption of sugary foods and drinks increases the likelihood of dental caries. However, it is of concern for the consequences of this strategy on oral health - that people with HD are encouraged to consume a diet high in sugar. Foods that are high in sugar often contain 'empty calories', and there is an association in people with HD with nutritional deficiency [16].

\section{- Percutaneous endoscopic gastrostomy}

In the later stages of $\mathrm{HD}$, a decision may be made to feed via PEG. The decision to insert a PEG may be dependent on a number of factors including:

- An advance directive regarding PEG insertion

- Dysphagia, which poses a significant risk of aspiration pneumonia

- Dysphagia, which significantly limits the calorific intake

It should additionally be recognized that a person with a PEG may also consume some oral foodstuffs providing it is not an aspiration risk.

There is very little literature about the oral health of people fed via PEG. Historical experimental research on animals helped to demonstrated that 'tube-fed' animals experienced less dental caries [17]. This view has been upheld by recent research that has shown that 'tube feeding had a remarkably lower odds ratio for new dental caries relative to regular meals' [18].

Several papers have reported a higher rate of plaque and calculus accumulation in people fed via PEG [19], but there have been no papers published that have specifically investigated periodontal disease in this group. However, it is widely known that gingivitis is a reversible inflammatory reaction of the gingiva to plaque accumulation, and that gingivitis precedes periodontitis. Not all gingivitis cases will develop into periodontitis - approximately $10 \%$ of individuals are highly susceptible and $10 \%$ are highly resistant to periodontitis. The difference in susceptibility is for the most part attributed to genetic factors [20].

The presence of dental plaque is the principle causative factor in the etiology of periodontal disease. It could, therefore, be suggested that this group of individuals who have shown a higher reported rate of plaque and calculus accumulation will show a higher prevalence of periodontal disease. It is clear that this is an area that deserves more research, particularly to clarify the exact prevalence of periodontal disease in those people who are fed via PEG.

\section{- Medication}

An assessment by the EHDN found that approximately $84 \%$ of HD registered patients receive symptomatic treatment [21]. The indications for prescribed medications were: 
- Depression, 50\%

- Chorea, 28\%

- Irritability and aggression, 13\%

- Sleep disturbance, 9\%

- Rigidity, apathy, mania, obsessive-compulsive disorders, $<2 \%$

The drugs prescribed varied depending upon the country; in the UK, citalopram, tetrabenazine, olanzapine and clonazepam are frequently prescribed. Citalopram is the single most prescribed antidepressant (41\%), and olanzapine was the neuroleptic chosen in $55 \%$ of cases in the UK. A common side-effect of these two medications is xerostomia [22,23]. Xerostomia has also been a reported as a sideeffect of tetrabenazine [24]. Xerostomia presents a significant problem in maintaining good oral health. Its presence is associated with a high prevalence and incidence of caries in several papers, and has additionally been associated with tooth loss and periodontitis [25].

A study of the oral health of a group of individuals with mid-late-stage $\mathrm{HD}$ in a specialized residential care facility found that the majority (33/35) of individuals were prescribed one or more medications with the potential to cause xerostomia as an undesirable side effect [8]. The average number of medications prescribed that can cause xerostomia as a side effect was 3.89, ranging from none to seven per patient. This study also noted further medications prescribed to these individuals with the potential to cause undesirable oral side effects. Gingival enlargement is a known side effect of sodium valproate and sertraline. Glossitis is an oral side effect of olanzipine, diazepam, sertraline venlafaxine and temazepam.

While it is appreciated that such medications may be important in the management of HD their implications for oral health present yet another challenge for preventing dental disease and maintaining good oral healthcare. It is a continuing concern to the dental profession that liquid medicines may contain cariogenic sweeteners. For some people with HD the oral intake of a variety of liquid medications on a frequent daily basis, if sugar containing, is another detrimental factor in preserving the dentition.

\section{- Oral hygiene}

A study of the oral health of a group of individuals with mid-late-stage HD with teeth in a specialized residential care facility found that these individuals had more plaque than the age-matched general population [8]. All of these individuals had gingival inflammation. Some of these individuals provided their own mouth care, and some required assistance with mouth care.

Self-provided oral hygiene may be poor in this group due to:

- Apathy and poor self care [26] - toothbrushing may not be a priority and their interest in hygiene diminishes as the disease progresses [7,21,27];

- Impairment of voluntary motor function [26] and impaired manual dexterity [7]. Toothbrushing may be difficult and inadequately performed due to movements and 'clumsiness'.

As the disease progresses people with HD become fully dependant on carers for activities of daily living, which includes oral care. Several papers have elucidated barriers to the provision of mouth care provided by a third party including:

- Lack of training [28,29]

- Time constraints [28,30,31]

- Lack of knowledge about oral care/disease [28-30,32]

- Lack of oral care supplies [30]

- Uncooperative residents/patients [30,31]

- Lack of staff [30]

- Need for/lack of an oral assessment tool [29,32]

- Low priority duty [29,31]

- Unrewarding/unpleasant task [29,31]

- Fear of causing pain [33]

- Use of ineffective mouth care equipment, for example, foam swabs instead of toothbrush [23]

These barriers may lead to the inadequate provision of mouth care and subsequently poor oral health. Although registered nurses (RNs) often receive some mouth care training, RNs were unlikely to provide mouth care. Mouth care is often delegated to healthcare assistants or their equivalent [31]. It is therefore possible that mouth care is performed by providers who receive no formal training in this field.

\section{- Access to \& provision of dental care}

There are many well recognized barriers of access to dental care for people with disabilities, such as: 
- Access to the dental surgery - such as the availability of transport to and from the building [4], wheelchair access [34]

- Financial constraints [4,35]

- Dentist's lack of experience treating people with disability [35]

- Caregivers' priorities for care [35]

People with HD may experience additional barriers to the receipt of dental care:

- Communication difficulties [27];

- Lack of dental professional's knowledge about $\mathrm{HD}$ and consequential reluctance to provide dental care [36];

- Difficulties providing treatment to patients with overt choreic movements $[4,27,36]$;

- Apathy and resentment in the patient and/or family [4]. Following diagnosis, people with HD may lose self-interest and may choose not to attend dental appointments or look after their oral health. An individual with HD may resent family members who may or may not have $\mathrm{HD}$ and complex psychological and behavioral issues can lead to the subsequent breakdown of family units. Conversely, family members may be the prime carers, but may also become apathetic towards or resent the individual with HD. The family carer may not want to or be able to provide the mouth care required for that individual, or assist them to their dental appointments as would be necessary to help maintain good oral health. An additional reason for family resentment in relation to dental care may be because of the inability of the dental treatment proposed to meet the expectations of the patient and/or caregivers. For example, difficulty or impossibility in providing advanced restorative care (crowns, bridges, implants);

- Ability to tolerate dental treatment due to involuntary movements $[4,36]$.

Regardless of these barriers, it is entirely feasible to provide a wide range of dental care to people with HD. Bradford et al. performed complex endodontics under local anesthetic alone: it was noted that the choreic movements prevented the full use of the dental operating microscope and the length of appointments were limited, however treatment was successful [36]. There have been several papers that have discussed the successful use of general anesthesia and conscious sedation to provide dental treatment for patients with HD who are unable to accept treatment under local anesthesia. Several anesthetic techniques have been described in the literature, including the uneventful use of isoflurane [7], succinylcholine [37], mivacurium [37,38], forane [37], nitrous oxide [37], sevoflurane $[38,39]$ and propofol $[39,40]$. General anesthesia has been used for a range of dental treatment: full mouth extractions, restorations, implant insertion and endodontics [7,27,37,39,40]. In one case, dental treatment was completed over five continuous hours of anesthesia [37].

Substantial consideration must be taken before general anesthesia is contemplated for HD-affected patients. It is more likely that treatment under general anesthesia is necessary in the later stages of the disease when there is a higher risk of complication. Dysphagia increases the risk of aspiration in the immediate postoperative period [7] and these patients are often malnourished [27,41] and dehydrated [27]. Certain anesthetic agents are avoided, anticholinergic drugs may increase choreic movements [7], and sodium thiopental has been reported to cause prolonged apnea.

Dental treatment under conscious sedation with intravenous midazolam has been recently used as an alternative to general anesthesia. It can be used effectively, often in small, titrated doses, to control movements and allow effective dentistry $[4,7,42,43]$. It has been suggested that conscious sedation can help to manage swallowing problems and reduce the risk of aspiration [4]. Cannulation may be difficult and may preclude a patient from receiving conscious sedation if movements are so great that intravenous access cannot be secured [7]. In such cases the use of oral or transmucosal (particularly intranasal) sedation may facilitate cannulation $[42,43]$. However, if sedation is not appropriate effective general anesthesia with a gaseous induction may be the only treatment option. One paper has described the use of diazepam administered through a patient's gastrostomy tube to facilitate dentistry [44].

\section{- Additional dental considerations}

Trauma

People with HD often display a disturbance in gait, can lose balance and are more susceptible to falls [7]. There has been one reported case of a fractured mandibular condyle following a fall 
where initial impact was the chin [45]. It should be noted that this group are prone to trips and falls, which can result in facial injuries.

\section{Prostheses}

Dentures become increasingly difficult to retain as the disease progresses due to xerostomia and reduced muscular control $[7,27,46]$. It is therefore important to retain functional teeth for as long as possible. Should a denture be considered a careful risk-benefit assessment must be made taking into account the possible instability and likelihood of airway obstruction.

\section{Bruxism}

One paper reported three cases of severe bruxism, which increased in severity alongside the progression of their HD. The bruxism resulted in severe tooth wear and jaw pain [45]. Others have also reported on its occurrence [47].

\section{A guideline for care}

The overall strategy for oral healthcare is based on the principle of achieving a disease-free, painfree and safe mouth. We seek to provide care and treatment that is no less a standard than that provided to an individual that does not have HD. Such care and treatment should take into account the health and safety of each individual within the context of their status.

HD provides particular challenges for oral healthcare. As with all aspects of healthcare, prevention should be introduced before the onset of the condition where possible. Some adults with the condition choose not to be formally diagnosed, and the lack of early identification of affected individuals may not allow for the instigation of early oral health prevention. In addition, the provision of dental treatment may be difficult because of the clinical features of HD - particularly the choreic movements. Such treatment difficulties make the need for prevention even more significant. For these reasons the importance of assessment and practical treatment planning cannot be overemphasized.

When planning and carrying out restorative care for people in the early stages careful attention should be given to the fact that the effective provision of oral hygiene, and therefore maintenance of advanced restorations, that is, crowns and bridges, may not be possible for those who will move into the advanced stages of the condition. Such issues may provide more difficulties for the dental practitioner and for the patient. A more pragmatic approach to treatment may well be in the interests of the patient, albeit not necessarily meeting patient and or relatives' expectations.

\section{- The stages of HD}

There have been several attempts to define the stages of progression of HD [48]. Current research may provide further options [49]:

- Early-stage disease cannot be defined as the time of diagnosis since diagnosis can be made at variable times. Most individuals present with early neurological and psychiatric features, which may have started to cause difficulties at work and home;

- Mid-stage disease would be seen to have been reached when the affected person has to cease work, or at least change employment to a post that is less challenging. Executive function decline and some cognitive deficit would be demonstrated. Involuntary movements would be obvious but it is most likely that individuals would still be able to feed themselves;

- Late-stage disease occurs when employment becomes impossible, the individual is no longer able to live independently, self care ceases and cognitive decline is obvious. Some patients maintain the ability to feed themselves to a late stage, but swallowing difficulties and involuntary movements create increasing difficulties.

It is recognized that HD is a continuum of varying clinical features. To interpret this into practical care and relate to the needs of the individual it is suggested that for the purpose of these guidelines the condition of HD is described by early, mid and late stages. Different authorities divide HD by three or five stages [48].

\section{- Relation to oral healthcare}

It is important to work with the family and carers as well as the affected individual and to provide as much choice as possible for care to each individual. Much of the care suggested will relate to the dependence on others for support to the person with HD. For this reason the guidelines will relate to the degree of dependence on care and services of that individual throughout the progression of their condition. Thus at the early stage the individual will be minimally dependent on others for support, he/she will be 
able to carry out good and effective oral hygiene and will accept all dental procedures with local anesthetic within primary care. At the middle stage, dependency will increase. The individual may still be able to carry out oral hygiene but will need occasional support (occasional physical help) for this process. Oral examination and treatment will be accepted using local anesthesia in the conventional manner; however, some more complex procedures may need to be carried out using sedation or general anesthesia. At the third stage, dependence will be at the highest level. Effective oral hygiene cannot be maintained by the individual alone who must have regular and effective daily physical support with this task. Oral examination is difficult and only the simplest treatment can be carried out with local anesthetic. The use of conscious sedation or general anesthesia would be the most appropriate option for treatment if suitable.

\section{Oral care regime \\ - Preventive care}

Much of the literature about HD and oral health discusses the importance of prevention. Boyle et al. make the point that at-risk families should be educated about the implications for oral health and encouraged to seek out regular care [7]. At-risk families and in particular newly diagnosed individuals should be referred at an early stage to a dental provider experienced in the condition. A thorough, preventative regime can then be implemented to reduce the risk of oral diseases and their sequelae as the disease progresses. Good oral health has positive benefits for health, dignity and self-esteem, social integration and general nutrition [5]. While people with HD are generally encouraged to eat a highly cariogenic diet and take medications that cause xerostomia to control or reduce their symptoms, the oral health of people with $\mathrm{HD}$ is at risk. Without multidisciplinary and interdisciplinary team working that includes a dental input, this risk is unlikely to change. The need for the implementation of an oral health program as early as possible cannot be over-emphasized. This should include appropriate preventive measures with close support from a hygienist. Sound guidance on good oral care is additionally available from the HD Association [104]. To further emphasize its importance a practical useful document is available online (www.FUtUREMEDicine.COM/Doi/ SUPpL/10.2217/NMT.11.68).

\section{- Assessment}

It is important that essential information about oral healthcare is available as soon as possible from non-dental agencies (medical, nursing, social care staff). An oral health assessment must also be carried out by a dental practitioner specializing in the care of adults with disability, preferably experienced in the dental care and management of people with HD. In the early stages of this condition patient involvement in treatment planning and long-term care is essential. This should include a discussion about the patient's expectations for their oral health and treatment. Individuals with $\mathrm{HD}$ may be encouraged to make advance decisions as is the case with other areas of their life [102]. This would then facilitate long-term planning of oral care between the individual and dental surgeon.

\section{- Treatment}

Provision of treatment should be appropriate to each individual case. This may include restorative care with local anesthetic, conscious sedation and/or general anesthetic. Careful consideration must be given before providing advanced restorative care as this may well be detrimental to the long-term patient care. Case studies form the bulk of literature written about HD and oral health. Although complex dentistry, including endodontics and implantology, has been described, long-term follow-up of these complex treatments have not been assessed nor how the oral health of these individuals is maintained as the disease progresses. In view of the fact that implants are becoming more a part of the provision of dental care, careful consideration should be given to their advantages and disadvantages. Ideally assessment should be provided by a specialist implantologist. A more pragmatic approach is to provide a more easily maintained dentition with good sound restorations following the onset of advanced HD. The provision of removable prostheses may be appropriate in the early stages, however, if provided it is essential that regular assessment be made of the potential risk that a denture may provide to airway obstruction. As such, all removal appliances should be clearly marked with radiopaque inserts.

The maintenance of good oral healthcare is very important for such patients and therefore regular and frequent oral assessments by an experienced practitioner are recommended. 
Summary guidelines

- Early stage/phase 0-2

Prevention

- Initial thorough examination (including radiographs and photography as appropriate) to obtain a baseline for future planning and to help with patient motivation. Review on a regular 6-monthly basis or as required

- Oral hygiene instruction with the use of adjunctive oral hygiene aids where appropriate

- Individual assessment for the use of the most appropriate form of fluoride, for example, high fluoride toothpaste, chlorhexadine and fluoride toothpaste [105], fluoride mouthwash

- Preventative advice to improve understanding of the significance of maintaining good oral health and the importance of smoking cessation

- Dietary advice to limit exposure to sugary foods and snacks. This should involve liaison with dieticians (see Dietician's Guidelines)

- Discussion of long-term care needs/treatment needs (future wishes)

\section{Restorative treatment}

- Restorative treatment should be high quality and low maintenance

- Maintenance of existing dentition by sound restorations, endodontics and prosthodontics

- Identify key teeth (e.g., canines, molars and occluding pairs) and restore to function

- Advanced restorative treatment planned so that, when the individual can no longer provide oral self-care, a caregiver is prepared and able to take on this role

- Use of local anesthetic with sedation as appropriate. Sedation may be administered orally/transmucosally, (intranasal/buccal), intravenously or inhalation sedation using nitrous oxide

- Use of radio-opaque identification on acrylic dentures

- Extraction of any unrestorable teeth

\section{- Middle stage/phase 2-4}

Prevention

- As above with more frequent (e.g., 3-4 monthly) reviews

- Use of fluoride varnish
- Introduction of oral hygiene aids, for example, Collis-curve toothbrush, Dr Barman's Superbrush, enlarged and/or weighted handles/grips, wrist weight cuffs, mouth angle expanders

- Oral hygiene instruction for carers using written instructions and/or pictures and other aids (e.g., DVDs)

- Consider introduction of a saliva substitute

Restorative treatment

- Delivery of treatment becomes more challenging

- If caries is active consider a shortened dental arch to provide a more easily maintained, functional dentition

- Treatment in a semi-reclined position with the use of support cushions help to reduce involuntary movements and relax body position

- Treatment becomes more likely to require the use of conscious sedation techniques, including oral/transmucosal sedation, intravenous sedation, or general anesthesia for delivery of care

- Frequent re-assessment of oral care plan, as per clinical need

- Consider patients capacity to consent to treatment and the need for consultation regarding all aspects of treatment

- Provision of simple and easily maintained dental treatment

\section{- Late stage/phase 4-5}

Prevention

- Daily oral care will be carried out by carers supported by regular input from dental professionals

- Written instructions and use of pictures as an aid to oral hygiene and to demonstrate preventive measures

- Regular swabbing of mouth with water or saliva substitutes (cooking oils may help) to reduce effects of xerostomia. This is especially required before meals and at night time

- Avoid mouthwash, which may pose an aspiration risk 


\section{Restorative treatment}

- Any interventive treatment is likely to require conscious sedation or general anesthesia. The patient's medical condition will be compromised so liaison with physicians and anesthetists will be required to help assess risk and palliative management

- If edentulous or significant tooth loss, do not consider the provision of removable prostheses, which may pose an aspiration risk

- The patient may be fed via PEG so emphasis should be placed on maintenance of a healthy and comfortable mouth

- When dental treatment using conscious sedation and general anesthesia is no longer appropriate, dental interventions should be as noninvasive as possible, for example, using Carisolv $^{\circledR}$ for caries removal, atraumatic restorative techniques, such as glass ionomer cement restorations

- Ensure a safe oral environment. For example, extract mobile teeth to prevent the risk of inadvertent inhalation

- Otherwise sound and periodontaly secure teeth may require extraction to prevent lip, tongue or cheek soft-tissue trauma resulting from uncontrolled movement or biting

- Balance the benefits of providing treatment against the difficulties in terms of co-operation, consent, and restraint. No treatment may be the appropriate option in palliative care.

An example of an information leaflet for carers is provided in the Supplementary brochure (wWw.FUTUREMEDICINE.COM/DOI/SUPPL/10.2217/NMT.11.68).

\section{Future perspective}

The oral healthcare of a person with HD should be based on effective, appropriate and continuing preventive care. Careful planning and provision of treatment needs to be implemented by clinicians experienced in the care of this patient group. Dental treatment must be appropriate to the stage of the condition. It should also be mindful of factors that relate to the progression of this condition that have an oral implication (e.g., risk of aspiration).

Central to every aspect of effective oral healthcare is the need to work as part of an interdisciplinary team. These core principles can only be developed through education, training and research. More dental care professionals need to be exposed to and involved in this group in a mentored and structured learning process. This can take place through postgraduate courses and be part of undergraduate curriculum. The courses should include other health professionals and caregivers to demonstrate the importance of multidisciplinary work. The specialty of special care dentistry is becoming more recognized for its valuable contribution within the dental profession through international (International Association for Disability and Oral Health) and national (e.g., British Society for Disability and Oral Health) bodies. More dentists working within this specialty will have experience in the care and treatment of people with HD.

There is an urgent need for practically based research that focuses on the needs of the individual, their family and caregivers. In addition research should be concerned with the effectiveness of preventive and treatment strategies that are tailored to the developing stages of the condition.

These guidelines present both a focus of this condition to the attention of the dental profession and a source of support for implementing good oral healthcare. The implementation of these guidelines should be an important task for dentists working in the area of disability and oral health.

The brochure/information leaflet is included as it serves to emphasize the importance and value of other health professionals and caregivers in being involved in oral healthcare. It also provides useful information for those individuals with HD.

\section{Acknowledgements}

The authors would like to acknowledge the contributing members of the European Huntington's Disease Network (EHDN) Standards of Care Dental Care Group to the writing of the Guideline document.

\section{Financial \& competing interests disclosure}

The EHDN had no role in study design, data collection and analysis, decision to publish, or preparation of the manuscript. The authors have no relevant affliations or financial involvement with any organization or entity with a financial interest in or financial conflict with the subject matter or materials discussed in the manuscript. This includes employment, consultancies, honoraria, stock ownership or options, expert testimony, grants or patents received or pending, or royalties.

No writing assistance was utilized in the production of this manuscript. 


\section{References}

Papers of special note have been highlighted as:

- of interest

- of considerable interest

1 Huntington's Disease (2nd Edition). Harper PS (Ed.). WB Saunders Company Limited, PA, USA (1996).

2 Quarrell OW, Rigby AS, Barron L et al. Reduced penetrance alleles for Huntington's disease: a multi-centre direct observational study. J. Med. Genet. 44(3) e68 (2007).

3 MacGiolla P, Guerin S, Nunn J. Train the trainers: a randomized, controlled trial of a multi-tiered health promotion programme to improve the oral health related knowledge, attitude, self-efficacy and behaviour of staff working with people with intellectual disability. J. Disabil. Oral Health 11(3), 107 (2010).

4 Lewis D. Fiske J. Dougall A. Access to special care dentistry, part 7. Special care dentistry services: seamless care for people in their middle years-part 1. Br. Dent. J. 205(6), 305-317 (2008).

- Discusses issues associated with access to dental care for people with disability.

5 Fiske J, Frenkel H, Griffiths J, Jones V. British Society of Gerodontology \& British Society for Disability and Oral Health: Guidelines for the development of local standards of oral health care for people with dementia. Gerodontology 23(Suppl. 1), 5-32 (2006).

6 Rada RE. Oral health care for the individual with Huntington's disease. In: Huntington's Disease: Etiology and Symptoms, Diagnosis and Treatment. Visser TJ (Ed.). Nova Science Publishers, NY, USA, 87-98 (2010).

7 Boyle CA, Frolander C, Manley MCG Providing dental care for patients with Huntington's disease. Den. Update 35(5), 333-336 (2008).

-1 Presents various treatment modalities for the care of people with Huntington's disease.

8 Lane H. The Oral Health and Oral Health Care of a Group of People with Huntington's Disease. MSc Thesis University of London (2009).

-E Presents a study examining the oral health and describing the dental care carried out in the study for people with Huntington's disease. An extensive review of the literature.

9 Gustaffson BE, Quensel CE, Lanke LS et al. The Vipeholm dental caries study. The effect of different levels of carbohydrate intake on caries activity in 436 individuals observed for five years. Acta Odontol. Scand. 11, 232-364 (1954).
10 Newbrun E. Frequent sugar intake - then and now: interpretation of the main results. Scand. J. Dent. Res. 97, 103-109 (1989).

11 Alvarez JO, Navia JM. Nutritional status, tooth eruption, and dental caries: a review. Am. J. Clin. Nutr. 49, 417-426 (1989).

12 Mobley CC. Nutrition and dental caries. Den. Clin. N. Am. 47, 319-336 (2003).

13 Kagel MC, Leopold NA. Dysphagia in Huntington's disease: a 16-year retrospective. Dysphagia 7, 106-114 (1992)

14 Hamakawa S, Koda C, Umeno H et al. Oropharyngeal dysphagia in a case of Huntington's disease. Auris Nasus Larynx 31, 171-176 (2004).

15 Leopold NA, Kagel MC. Dysphagia in Huntington's disease. Arch. Neurol. 42, 57-60 (1985).

16 Lanska DJ, Lanska MJ, Lavine L, Schoenberg BS. Conditions associated with Huntington's disease at death. A case-control study. Arch. Neurol. 45, 878-880 (1998).

17 Kite OW, Shaw JH, Sognnaes RF. The prevention of experimental tooth decay by tube-feeding. Nutrition 42(1), 89-105 (1950).

18 Idaira Y, Nomura Y, Tamaki Y et al. Factors affecting the oral condition of patients with severe motor and intellectual disabilities. Oral Dis. 14, 435-439 (2008).

19 Jawadi AH, Casamassimo PS, Griffen A, Enrile B, Marcone M. Comparison of oral findings in special needs children with and without gastrostomy. Pediatr. Dent. 26(3) 283-288 (2004).

20 Tatakis DN, Kumar PS. Etiology and pathogenesis of periodontal diseases. Den. Clin. N. Am. 49, 491-516 (2005).

21 Priller J, Ecker D, Craufurd D. A Europewide assessment of current medication choices in Huntington's disease (Letter). Mov. Disord. 23(12), 1788 (2008).

22 British National Formulary (Volume 57). BMJ Group, London, UK, and RPS Publishing, London, UK, (2009).

23 Pearson LS, Hutton JL. A controlled trial to compare the ability of foam swabs and toothbrushes to remove dental plaque. J. $A d v$ Nurs. 39(5) 480-489 (2002).

24 Fasano A, Cadeddu F, Guidubaldi A. The long-term effect of tetrabenazine in the management of Huntington disease. Clin. Neuropharmacol. 31(6), 313-318 (2008).

25 Papas AS, Singh M, Harrington BS, Ortblad $\mathrm{K}$, de Jager M, Nunn M. Reduction in caries rate among patients with xerostomia using a power toothbrush. Spec. Care Dentist. 27(2), 46-51 (2007).
26 Huntington's Disease 3rd Edition. Bates G, Harper P, Jones L (Eds). Oxford University Press, Oxford, UK, (2002).

27 Jackowski J, Andrich J, Kappeler H, Zollner A, Johren P, Muller T. Implant-supported denture in a patient with Huntington's disease: interdisciplinary aspects. Spec. Care Dentist. 21(1), 15-20, (2001).

28 Weeks JC, Fiske J. Oral care of people with a disability: a qualitative exploration of the views of nursing staff. Gerodontology 11(1), 13-17 (1994)

29 Gillam JL, Gillam DG. The assessment and implementation of mouth care in palliative care: a review. J. R. Soc. Promot. Health 126, 33-37 (2006).

30 Coleman P, Watson NM. Oral care provided by certified nursing assistants in nursing homes. J. Am. Geriatr. Soc. 54, 138-143 (2006).

31 Wardh I, Andersson L, Sorensen S. Staff attitudes to oral care. A comparative study of registered nurses, nursing assistants and home care aides. Gerodontology 14(1), 28-32 (1997).

32 Adams R. Qualified nurses lack adequate knowledge related to oral health, resulting in inadequate oral care of patients on medical wards. J. Adv. Nurs. 24, 552-560 (1996).

33 Berry AM, Davidson PM. Beyond comfort: oral hygiene as a critical nursing activity in the intensive care unit. Intensive Crit. Care Nurs. 22, 318-328 (2006).

34 Dougall A, Fiske J. Access to special care dentistry, part 1. Access. Br. Dent. J. 204(11), 605-616 (2008).

35 Fiske J, Shafik HH. Down's syndrome and oral care. Dent. Update 28, 148-156 (2001).

36 Bradford H, Britto L, Leal G, Katz J. Endodontic treatment of a patient with Huntington's disease. J. Endod. 30(5), 366-369 (2004).

- Case study of restorative care for an adult with Huntington's disease.

37 Rada R. Comprehensive dental treatment of a patient with Huntington's disease: literature review and case report. Spec. Care Dentist. 28(4), 131-135, (2008).

38 Nagele P, Hammerle AF. Sevoflurane and mivacurium in a patient with Huntington's chorea. Br. J. Anaesth. 85(2), 320-321 (2000).

39 Cangemi CF Jr, Miller RJ. Huntington's disease: review and anesthetic case management. Anesth. Prog. 45(4), 150-153 (1998).

40 Soar J, Matheson KH. A safe anaesthetic in Huntington's disease. Anaesthesia 48(8) 743-744 (1993). 
41 Nandita K, Jatin L, Sarla H. Anesthetic management of a patient with Huntington's chorea. (Letter) Neurol. India 56(4), 486-487 (2008).

42 Manley MCG. Neuro-disability and oral care (Video presentation, available on request from MCG Manley). Br. Soc. Disabil. Oral Health (2008).

43 Manley MCG, Ransford NJ, Lewis DA, Thompson SA, Forbes M. Retrospective audit of the efficacy and safety of the combined intranasal/intravenous midazolam sedation technique for the treatment of adults with learning disability. Br. Dent. J. 205(2), E3 (2008).

- An audit of the use of conscious sedation using intranasal and intravenous sedation for people with challenging behavior. A very valuable technique in the treatment of people with Huntington's disease.

44 da Fonseca MA, Walker PO. Dental management of a child with Huntington's disease: case report. Spec. Care Dentist. 13(2), 71-73 (1993).

45 Starck WJ, Morrissette MP, Chewning LC. Treating a mandibular condylar fracture in a patient with Huntington's disease. J. Am. Dent. Assoc. 123(9), 52-58 (1992).

46 Kieser J, Jones G, Borlase G, MacFadyen E. Dental treatment of patients with neurodegenerative disease. $N Z$ Dent. J. 95(422), 130-134 (1999).
47 Tan E-K, Jankovic J, Ondo W. Bruxism in Huntington's disease. (Letter). Mov. Disord. 13(1), 171-173 (2000).

48 Shoulson I, Fahn S. Huntington disease: clinical care and evaluation. Neurology 29(1),1-3 (1979).

49 Paulsen JS, Langbehn DR, Stout JC et al. Detection of Huntington's disease decades before diagnosis: the Predict-HD study. J. Neurol. Neurosurg. Psychiatry 79(8), 874-880 (2008).

\section{- Websites}

101 European Huntington's Disease Network. www.euro-hd.net

102 Huntington's disease association fact sheet 7 : Huntington's disease and diet. www.hda.org.uk/download/fact-sheets/HDDiet.pdf

103 Australian Huntington's Disease Association (NSW) Inc.

www.ahdansw.asn.au/information/nutrition. html

104 Huntington's Disease association fact sheet 8 : the importance of dental care.

www.hda.org.uk/download/fact-sheets/HDDental-Care.pdf

105 Sunstar Gum.

www.sunstargum.com/index.php?id=4084
Contributing members

- UK

- Graham Manley

- Helen Lane

- David Wong

- Rhona Brown

- Aniko Balazs

- Alison Hamilton

- Daniela Rae

- Sheila Simpson

- The Netherlands

- Peter van Splunter

- Sweden

- Annette Carlsson

- Bitte Ahlborg

- Åsa Mårtensson

- Monica B Nilsson

- Czech Republic

- Pavla Sasinkova 